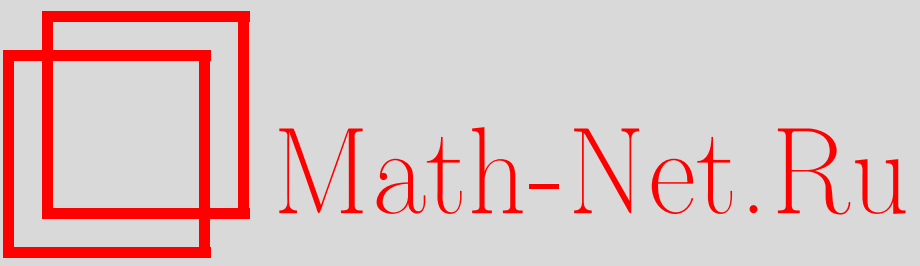

Б. А. де Монвель, М. В. Щербина, О свободной энергии в двумерной $U(n)$ калибровочной теории поля на сфере, ТМФ, 1998, том 115, номер 3, 389-401

DOI: https://doi.org/10.4213/tmf881

Использование Общероссийского математического портала Math-Net.Ru подразумевает, что вы прочитали и согласны с пользовательским соглашением

http://www. mathnet.ru/rus/agreement

Параметры загрузки :

IP : 54.197 .217 .227

26 апреля 2023 г., 05:32:28 
ТЕОРЕТИЧЕСКАЯ

И МАТЕМАТИЧЕСКАЯ

ФИЗИКА

Том 115, № 3

июнь, 1998

(C) 1998 г. $\quad$ А. Буте де Монвель*, М.В. Шербина ${ }^{\dagger}$

\title{
О СВОБОДНОЙ ЭНЕРГИИ В ДВУМЕРНОЙ $U(n)$-КАЛИБРОВОЧНОЙ ТЕОРИИ ПОЛЯ НА СФЕРЕ
}

\begin{abstract}
Математически строго получена статистическая сумма в двумерной $U(n)$-калибровочной теории поля в пределе $n \rightarrow \infty$. Полученные результаты и использованные методы стимулированы недавними исследованиями в теории случайных матриц в сочетании с традиционным аппаратом статистической механики.
\end{abstract}

\section{1. ВВЕДЕНИЕ}

Двумерная неабелева калибровочная теория поля $\left(\mathrm{QCD}_{2}\right)$ может служить полезным полигоном для изучения математической структуры физически аналогичных теорий в пространствах с подходящим числом измерений (струнные аспекты, фаза конфайнмента, поведение различных инвариантных величин и т.д.). Важным объектом в неабелевых теориях является статистическая сумма (а в более обшем случае математическое ожидание вильсоновских петель) на двумерных поверхностях. Эта функция является ядром (или его следом) уравнения теплопроводности на многообразии калибровочной группы, как это и ожидается из идеологии функционального интегрирования. Во многих интересных случаях можно явно выписать эти функции в терминах неприводимых представлений калибровочной группы. Это обстоятельство вызвало к жизни многочисленные исследования $\mathrm{QCD}_{2}$ и смежных сюжетов.

В [1] была проанализирована статистическая сумма, отвечающая $U(n)$-калибровочной теории в (планарном) пределе больщих $n$. Авторы привели аргументы в пользу того, что в этом пределе возникает фазовый переход третьего рода при изменении площади $A$. Поскольку константа связи входит в статистическую сумму только в комбинации $g^{2} A$, фазовый переход можно интерпретировать как определенное изменение асимптотического поведения (при больших $n$ ) в режиме сильной связи по сравнению с (тривиальным) режимом слабой связи. За ссылками и обсуждением QCD-аспектов фазового перехода мы отсылаем читателя к работам [1-3].

В настояшей статье мы даем строгий вьвод выражения для свободной энергии, найденного в [1]. Наш вывод базируется на традиционных идеях статистической механики.

\footnotetext{
* Лаборатория математической физики и геометрии Университета, Париж VII, Франция

${ }^{\dagger}$ Институт физики низких температур, Харьков, Украина
} 
Именно, мы используем эквивалентность канонического и большого канонического ансамблей, а также метод предельного среднего поля.

Статистическая сумма, найденная в [1], имеет вид суммы по всем неприводимым представлениям $R$ группы $U(n)$ :

$$
Z(n, A)=\sum_{R}(\operatorname{dim} R)^{2} \exp \left\{-\frac{A}{2 n} C_{2}(R)\right\}
$$

Здесь $\operatorname{dim} R$ - размерность представления $R, C_{2}(R)$ - собственное значение квадратичного оператора Казимира (лапласиана), а $A$ - плошадь сферы. Пользуясь стандартной параметризацией неприводимых представлений $U(n)$ посредством соответствующих весов, можем записать статистическую сумму (1.1) в следуюшем виде [1]:

$$
\begin{aligned}
Z(n, A)= & \frac{1}{n !} e^{-\frac{A}{24}\left(n^{2}-1\right)} \times \\
& \times \sum_{h_{1}, \ldots, h_{n}=-\infty}^{\infty} \exp \left\{-\frac{A n}{2} \sum_{i=1}^{n}\left(\frac{h_{i}}{n}\right)^{2}\right\} \prod_{1 \leqslant i<j \leqslant n}\left(h_{i}-h_{j}\right)^{2},
\end{aligned}
$$

где суммирование идет по всем целым числам $h_{1}, \ldots, h_{n}$. Легко увидеть, что с точностью до тривиального множителя

$$
\frac{1}{n !} e^{-\frac{A}{24}\left(n^{2}-1\right)}
$$

это выражение совпадает со статистической суммой унитарно-инвариантного ансамбля эрмитовых случайных матриц [4]

$$
\widehat{Z}_{n}=\int_{-\infty}^{\infty} \cdots \int_{-\infty}^{\infty} \exp \left\{-n \sum_{i=1}^{n} V\left(x_{i}\right)\right\} \prod_{1 \leqslant i<j \leqslant n}\left(x_{i}-x_{j}\right)^{2} \prod_{i=1}^{n} d x_{i}
$$

где $V(x)=A x^{2} / 2$. В самом деле, если заменить суммирование в (1.2) интегрированием, мы получим выражение (1.3). Асимптотическое поведение $\hat{Z}_{n}$ было найдено физиками много лет назад (см. $[5,6])$. Математически строго оно было доказано в [7]. Тем не менее прямое применение этих методов приводит к правильному выражению (1.2) только при малых $A$ (фаза слабой связи). Таким образом, мы встречаем здесь фазовый переход нового типа, которого нет в непрерывной версии (1.2), т.е. в матричной модели. Чтобы понять математическую природу этого фазового перехода, рассчитаем "игрушечную" статистическую сумму

$$
\begin{aligned}
& \hat{Z}_{n}^{a}=\int_{x_{i} \neq x_{j}} \exp \left\{-n \sum_{i=1}^{n} V\left(x_{i}\right)\right\} \prod_{i=1}^{n} d x_{i}, \\
& Z_{n}^{a}=\sum_{h_{i} \neq h_{j}} \exp \left\{-n \sum_{i=1}^{n} V\left(h_{i}\right)\right\}
\end{aligned}
$$


из которой удалены “члены взаимодействия"

$$
\prod_{i<j}\left(h_{i}-h_{j}\right)^{2} \quad \text { и } \quad \prod_{i<j}\left(x_{i}-x_{j}\right)^{2},
$$

а также использованы более простые условия

$$
x_{i} \neq x_{j} \text { или } h_{i} \neq h_{j}, \quad i \neq j .
$$

Непрерьвная статистическая сумма (1.4) факторизуется:

$$
\hat{Z}_{n}^{a}=\prod_{i=1}^{n} \int e^{-n V\left(x_{i}\right)} d x_{i}
$$

Дискретную статистическую сумму $Z_{n}^{a}$, вообще говоря, нельзя записать в таком виде, т.к. даже асимптотически при больших $n$ равенство

$$
Z_{n}^{a}=\prod_{i=1}^{n} \sum e^{-n V\left(\frac{h_{i}}{n}\right)}
$$

справедливо только при некотором специальном выборе $V(x)$, который соответствует “фазе слабой связи”. Это явление объясняется тем, что в непрерывном случае условие (1.5) не влияет на интеграл, меж тем как в дискретном случае оно сушественно, в особенности в ситуации фазового перехода. Можно сравнить эту проблему с проблемой вычисления свободной энергии идеального бозе-газа, где разница между дискретным и непрерывным случаями (т.е. между суммированием и интегрированием) приводит к конденсации Бозе-Эйнштейна.

Таким образом, чтобы найти асимптотическое поведение $Z_{n}^{a}$, мы нуждаемся в технике, способной автоматически учесть условия (1.5). Такая техника хорошо известна в статистической механике. Она сводится к обычному методу доказывать эквивалентность большого канонического и канонического ансамблей, вводя химический потенциал. Этой техникой мы докажем следуюшую теорему, которая является строгой формулировкой главного результата [1].

ТеОРема 1. Рассмотрим дискретную статистическую сумму в виде

$$
Z_{n}=\sum_{h_{i} \neq h_{j}} \exp \left\{\sum_{i \neq j} \log \left|h_{i}-h_{j}\right|-\sum_{i=1}^{n} n V\left(\frac{h_{i}}{n}\right)\right\}
$$

где $V(x)$ удовлетворяет условию

$$
|V(x)|>(2+\epsilon) \log |x|
$$

Тогда существует предельная свободная әнергия

$$
f \equiv \lim _{n \rightarrow \infty} n^{-2} \log Z_{n}=\int \log \left|x-x^{\prime}\right| \rho(x) \rho\left(x^{\prime}\right) d x d x^{\prime}-\int \rho(x) V(x) d x,
$$


где плотность $\rho(x)$ однозначно определяется из следующих условий:

a)

$$
\rho(x) \geqslant 0, \quad \int \rho(x) d x=1
$$

б)

$$
\rho(x) \leqslant 1
$$

в) существует константа z такая, что

$$
\operatorname{supp} \rho(x) \in\{x: u(x) \geqslant-z\}
$$

$u$

$$
\rho(x)=1 \quad n p u \quad u(x)>-z .
$$

Здесь по определению

$$
u(x) \equiv 2 \int \log \left|x-x^{\prime}\right| \rho\left(x^{\prime}\right) d x^{\prime}-V(x) .
$$

ЗАМЕчАнИЕ. Легко показать, что свободную энергию $f$ и плотность $\rho(x)$ можно получить как решение следуюшей вариационной задачи:

$$
f=\sup _{\rho(x) \leqslant 1}\left\{\int \log \left|x-x^{\prime}\right| \rho(x) d x \rho\left(x^{\prime}\right) d x^{\prime}-\int V(x) \rho(x) d x\right\},
$$

где супремум берется по всем плотностям $\rho(x)$, удовлетворяющим условию "б”. С другой стороны, решение непрерывной проблемы (1.3) есть

$$
\begin{aligned}
\hat{f} & \equiv \lim _{n \rightarrow \infty} n^{-2} \log \hat{Z}_{n}= \\
& =\sup _{\hat{\rho}(x)}\left\{\int \log \left|x-x^{\prime}\right| \hat{\rho}(x) d x \hat{\rho}\left(x^{\prime}\right) d x^{\prime}-\int V(x) \hat{\rho}(x) d x\right\}
\end{aligned}
$$

(доказательство и обсуждение см. в [7]), где супремум берется по всем плотностям без этого ограничения. Таким образом, в фазе слабой связи мы получаем одно и то же решение и для дискретного, и для непрерывного случаев. С другой стороны, мы находим разные решения проблемы (1.2) и (1.3) (в фазе сильной связи $(1.2))$, поскольку $\hat{\rho}\left(x_{0}\right)=1$ в некоторой точке $x_{0}$ для какой-то $A=A_{\mathrm{c}}$ и $A>A_{\mathrm{c}}$.

Чтобы найти $\rho(x)$ (или $\hat{\rho}(x))$ в фазе слабой связи в простейшем случае, когда $V(x)$ является четной функцией только с одним минимумом (например, $V(x)=x^{2}$ ), мы должны решить сингулярное интегральное уравнение

$$
V^{\prime}(x)=2 \int_{-a}^{a} \frac{\rho(y) d y}{x-y} .
$$


Это уравнение имеет ограниченное решение для любых $a$ (см. [8]). Константа $a$ определяется нормировочным условием "a". В фазе сильной связи функция $\rho(x)$ удовлетворяет следующим условиям:

$$
\begin{gathered}
\rho(x)=0, \text { если }|x| \geqslant a, \\
0 \leqslant \rho(x) \leqslant 1, \text { если } a>|x|>b, \\
\rho(x)=1, \text { если }|x| \leqslant b
\end{gathered}
$$

согласно условиям "a”, “б”, "в”. Поэтому нужно решить другое сингулярное уравнение (cp. с (1.15)):

$$
V^{\prime}(x)=2 \int_{-a}^{-b} \rho(y) d y\left(\frac{1}{x-y}+\frac{1}{x+y}\right)+2 \int_{-b}^{b} \frac{d y}{x-y}
$$

и найти $a, b$ из условия нормировки "а" и уравнения

$$
u(-b)=u(b)
$$

которое следует из условия "В" для функции $u(x)$ формулы (1.12).

В случае, если $V(x)$ имеет $m$ минимумов, носитель $\sigma$ плотности $\rho(x)$ может состоять из $m$ интервалов. Для каждого такого интервала $\left(a_{i}, a_{i+1}\right), i=1,3, \ldots, 2 k-1$, рассмотрим маленький интервал $\left(b_{i}, b_{i+1}\right) \subset\left(a_{i}, a_{i+1}\right)$, в котором $\rho(x)=1$, и найдем $\rho(x)$ на множестве

$$
\sigma^{\prime}=\bigcup_{i=1}^{2 k-1}\left(\left(a_{i}, b_{i}\right) \cup\left(b_{i+1}, a_{i+1}\right)\right)
$$

как решение сингулярного интегрального уравнения

$$
V^{\prime}(x)=2 \int_{\sigma} \frac{\rho(y) d y}{x-y} \text { при } x \in \sigma^{\prime} .
$$

Согласно терии сингулярных интегральных уравнений [8] это уравнение имеет ограниченное решение, если функция $V(x)$ удовлетворяет $2 k$ условиям на множестве $\sigma^{\prime}$. Это дает нам $2 k$ уравнений в концевых точках $a_{1}, \ldots, a_{2 k}, b_{1}, \ldots, b_{2 k}$. Другие уравнения можно найти из нормировочных условий "а" и из равенств

$$
\begin{array}{lll}
u\left(b_{1}\right)=u\left(b_{2}\right), & \ldots, & u\left(b_{2 k-1}\right)=u\left(b_{2 k}\right), \\
u\left(a_{2}\right)=u\left(a_{3}\right), & \ldots, & u\left(a_{2 k-2}\right)=u\left(b_{2 k-1}\right),
\end{array}
$$

которые вытекают из соотношений (1.10), (1.11). 


\section{2. ДОКАЗАТЕЛЬСТВА}

Заметим, что в силу леммы (см. ниже) сушествуют положительные числа $L>2$ и $\delta>0$ такие, что

$$
\left|\frac{1}{n^{2}} \log Z_{n}-\frac{1}{n^{2}} \log Z_{n}^{L}\right| \leqslant e^{-n \delta},
$$

где

$$
Z_{n}^{L}=\sum_{h_{i} \neq h_{j},\left|h_{j}\right| \leqslant n L} \exp \left\{\sum_{i \neq j} \log \left|h_{i}-h_{j}\right|-\sum_{i=1}^{n} n V\left(\frac{h_{i}}{n}\right)\right\} .
$$

Оказывается, что $Z_{n}^{L}$ можно рассматривать как статистическую сумму некоторой одномерной модели типа Изинга. Гамильтониан этой модели имеет вид

$$
\begin{aligned}
H(\nu)= & -\frac{1}{n} \sum_{r \neq r^{\prime},|r|<n L} \log \left|\frac{r-r^{\prime}}{n L}\right| \nu_{r} \nu_{r^{\prime}}- \\
& -\sum_{|r|<n L} V\left(\frac{r}{n}\right) \nu_{r}-\frac{1}{n} \sum_{|r|<n L}\left(\log \frac{1}{n L}+1\right) \nu_{r}
\end{aligned}
$$

где $\nu_{r}=0,1$ - “числа заполнения". Итак, имеем

$$
Z_{n}^{L}=\sum_{\left\{\sum \nu_{r}=n\right\}} \exp \{-n H(\nu)+n(n-1) \log L n+n(\log n L-1)\} .
$$

Чтобы освободиться от условия $\sum \nu_{r}=n$, введем в гамильтониан параметр $z$ ("химический потенциал”). Эта процедура вполне стандартна в статистической механике:

$$
H(\nu, z)=\sum_{|r|,\left|r^{\prime}\right|<n L} w\left(\frac{r-r^{\prime}}{n L}\right) \nu_{r} \nu_{r^{\prime}}+\sum_{|r|<n L}\left(V\left(\frac{r}{n}\right)-z\right) \nu_{r}+n z,
$$

где

$$
w(r)= \begin{cases}-n^{-1} \log |r|, & r \neq 0 \\ -n^{-1}(\log n L-1), & r=0\end{cases}
$$

Рассмотрим

$$
Z_{n}(z)=\sum_{\left\{\nu_{r}\right\}} \exp \{-n H(\nu, z)\}
$$

Теперь воспользуемся условием

$$
\left\langle\frac{1}{n} \sum_{|r|<n L} \nu_{r}\right\rangle_{H(z)}=1
$$

чтобы рассчитать z. Если мы докажем, что

$$
\left\langle\left(\frac{1}{n} \sum_{|r|<n L} \nu_{r}-1\right)^{2}\right\rangle_{H(z)} \rightarrow 0 \text { при } n \rightarrow \infty
$$


то (это также вполне обычно для статистической механики) мы сможем утверждать, чTO

$$
\lim _{n \rightarrow \infty}\left|\frac{1}{n^{2}} \log Z_{n}^{L}-\frac{1}{n^{2}} \log Z_{n}(z)-\log n L\right|=0
$$

Итак, чтобы рассчитать $Z_{n}$, нужно вычислить $Z_{n}(z)$, затем найти $z$ из уравнения $(2.7)$ и проверить (2.8). Чтобы сделать все это, воспользуемся методом среднего поля в форме, предложенной в [9] для аналогичных целей. Рассмотрим так называемый приближенный гамильтониан вида

$$
\begin{aligned}
H_{a}(\nu, c, z)= & 2 \sum_{|r|,\left|r^{\prime}\right|<n L} w\left(\frac{r-r^{\prime}}{n L}\right) \nu_{r} c_{r^{\prime}}-\sum_{|r|,\left|r^{\prime}\right|<n L} w\left(\frac{r-r^{\prime}}{n L}\right) c_{r} c_{r^{\prime}}+ \\
& +\sum_{|r|<n L}\left(V\left(\frac{r}{n}\right)-z\right) \nu_{r}+n z .
\end{aligned}
$$

Тогда получаем

$$
\begin{aligned}
H(\nu, z)= & H_{a}(\nu, c, z)+ \\
& +\sum_{|r|,\left|r^{\prime}\right|<n L} w\left(\frac{r-r^{\prime}}{n L}\right)\left(\nu_{r}-c_{r}\right)\left(\nu_{r^{\prime}}-c_{r^{\prime}}\right)=H_{a}+R .
\end{aligned}
$$

Согласно неравенству Боголюбова [10] имеем

$$
\frac{1}{n^{2}}\langle R\rangle_{H(z)} \leqslant \frac{1}{n^{2}} \log Z_{n}(z)-\frac{1}{n^{2}} \log Z_{n}(c, z) \leqslant \frac{1}{n^{2}}\langle R\rangle_{H_{a}(c, z)}
$$

где $Z_{n}(c, z)$ - статистическая сумма, отвечающая гамильтониану $H_{a}(c, z)$. Для любого $z$ выберем $c^{(n)}=\left\{c_{r}^{(n)}\right\}-$ точку минимума функции

$$
\Phi(c, z)=\frac{1}{n^{2}} \log Z_{n}(c, z)
$$

Поскольку в [7] было доказано, что

$$
w\left(\frac{r-r^{\prime}}{n L}\right)
$$

- это положительная матрица, функция $\Phi$ является "строго выпуклой” по оношению к $c$, $z$ (т.е. ее вторые производные по всем направлениям строго положительны). Кроме того, она растет при $c \rightarrow \infty$. Поэтому для всех $z$ функция $\Phi(c, z)$ принимает минимальное значение относительно $c$ в единственной точке $c^{(n)}$, которая является решением следующего уравнения:

$$
c_{r}^{(n)}=\left\langle\nu_{r}\right\rangle_{H_{a}(c, z)}=\frac{\exp \left\{n\left(u_{n}(r / n)+z\right)\right\}}{1+\exp \left\{n\left(u_{n}(r / n)+z\right)\right\}},
$$


где

$$
u_{n}\left(\frac{r}{n}\right)=-\sum_{\left|r^{\prime}\right|<L} w\left(\frac{r-r^{\prime}}{n L}\right) c_{r^{\prime}}^{(n)}+V\left(\frac{r}{n}\right)
$$

Следовательно, если мы положим $c=c^{(n)}$ в $(2.10)$ и воспользуемся тем фактом, что

$$
\left\langle\nu_{r} \nu_{r^{\prime}}\right\rangle_{H_{a}(c, z)}=\left\langle\nu_{r}\right\rangle_{H_{a}(c, z)}\left\langle\nu_{r^{\prime}}\right\rangle_{H_{a}(c, z)}\left(r \neq r^{\prime}\right)
$$

мы получим

$$
\begin{aligned}
\frac{1}{n^{2}}\langle R\rangle_{H(z)} & \leqslant \frac{1}{n^{2}} \log Z_{n}(z)-\frac{1}{n^{2}} \log Z_{n}(c, z) \leqslant \\
& \leqslant \frac{1}{n} w(0)=\frac{\log n L+1}{n}
\end{aligned}
$$

Благодаря положительности матрицы

$$
w\left(\frac{r-r^{\prime}}{n L}\right)
$$

левая часть этого неравенства положительна. Поэтому

$$
\lim _{n \rightarrow \infty}\left|\frac{1}{n^{2}} \log Z_{n}(z)-\min _{c} \frac{1}{n^{2}} \log Z_{n}(c, z)\right|=0
$$

и

$$
\left\langle\frac{1}{n} \sum_{|r|,\left|r^{\prime}\right|<n L} w\left(\frac{r-r^{\prime}}{n L}\right)\left(\nu_{r}-c_{r}\right)\left(\nu_{r^{\prime}}-c_{r^{\prime}}\right)\right\rangle_{H(z)} \leqslant \frac{\log n L+1}{n} .
$$

Как это доказано в [7], из последнего неравенства следует, что

$$
\left\langle\left(\frac{1}{n} \sum_{|r|<n L} \nu_{r}-\frac{1}{n} \sum_{|r|<n L} c_{r}^{(n)}\right)^{2}\right\rangle_{H(z)} \leqslant \text { const } \cdot n^{-1 / 2} \log ^{1 / 2} n .
$$

Итак, мы разрешим уравнение (2.7) и докажем (2.8), если выберем $z$, совместимое с уравнением

$$
\frac{1}{n} \sum_{|r|<n L} c_{r}^{(n)}=1
$$

Последнее равенство - это как раз уравнение для критической точки функционала $\Phi(c, z)$. Как отмечалось выше, этот функционал является “строго вьпуклым" относительно $c$ и растет при $z \rightarrow \infty$. Значит, сушествует единственная точка $z^{(n)}$, которая удовлетворяет условию (2.18). Согласно (2.9) и (2.12) имеем

$$
\lim _{n \rightarrow \infty}\left|\frac{1}{n^{2}} \log Z_{n}^{L}-\min _{c, z} \Phi_{n}(c, z)-\log n L\right|=0
$$


Теперь изучим $\min _{c, z} \Phi_{n}(c, z)=\Phi_{n}\left(c^{(n)}, z^{(n)}\right)$. Из (2.13) вытекает, что

$$
0 \leqslant c_{r}^{(n)} \leqslant 1 .
$$

Рассмотрим меры

$$
\mu^{(n)}(\Delta)=\frac{1}{n} \sum_{|r|<n L} c_{r}^{(n)} \chi_{\Delta}\left(\frac{r}{n}\right),
$$

где $\chi_{\Delta}(x)$ - характеристическая функция интервала $\Delta$. По теореме Хелли существуют подпоследовательность $\mu^{\left(n_{k}\right)}$, мера $\mu$ и константа $z$ такие, что $\mu^{\left(n_{k}\right)}(\Delta) \rightarrow \mu(\Delta)$ для некоторого $\Delta$ и $z^{\left(n_{k}\right)} \rightarrow z$ при $k \rightarrow \infty$. Поскольку $\mu^{\left(n_{k}\right)}$ имеет вид $(2.21)$, а $c_{r}^{\left(n_{k}\right)}$ удовлетворяют (2.18) и (2.20), предельная мера $\mu(d x)$ абсолютно непрерьвна, $\mu(d x)=\rho(x) d x$, и ее плотность $\rho(x)$ удовлетворяет условиям

$$
0 \leqslant \rho(x) \leqslant 1, \quad \int \rho(x) d x=1 .
$$

Пусть

$$
u(x)=2 \int \log \left|\frac{x-x^{\prime}}{L}\right| \rho\left(x^{\prime}\right) d x^{\prime}-V(x) .
$$

Поскольку $c^{(n)}$ задаются решением (2.13), имеем (см. (1.8)-(1.12))

$$
\operatorname{supp} \rho(x) \in\{x: u(x) \geqslant-z\}
$$

и

$$
\rho(x)=1, \text { если } u(x)>-z .
$$

Мы докажем сейчас, что условия (2.22)-(2.25) определяют функцию $\rho(x)$ однозначно. Для этого предположим, что сушествует другая функция $\rho_{1}(x)$, удовлетворяюшая тем же условиям (быть может, с другим $z=z_{1}$ ). Рассмотрим

$$
\begin{gathered}
u^{1}(x)=2 \int \log \left|\frac{x-x^{\prime}}{L}\right| \rho_{1}\left(x^{\prime}\right) d x^{\prime}-V(x) ; \\
d_{n}\left(\frac{r}{n}\right)=\rho_{1}\left(\frac{r}{n}\right)+n^{-1 / 2}\left(c_{r}^{(n)}-\rho_{1}\left(\frac{r}{n}\right)\right) ; \\
u_{n}^{1}\left(\frac{r}{n}\right)=-\sum_{\left|r^{\prime}\right|<L} w\left(\frac{r-r^{\prime}}{n L}\right) d_{n}\left(\frac{r^{\prime}}{n}\right)+V\left(\frac{r}{n}\right)= \\
=u^{1}\left(\frac{r}{n}\right)+n^{-1 / 2}\left(u\left(\frac{r}{n}\right)-u^{1}\left(\frac{r}{n}\right)\right)+O\left(n^{-1} \log n\right),
\end{gathered}
$$


где $u_{n}(r / n)$ определено в (2.14). Так как $\Phi_{n}(c, z)$ - выпуклая функция, а $\left(c^{(n)}, z^{(n)}\right)$ точка минимума, будем иметь для $n=n_{k}$

$$
\begin{aligned}
0 \leqslant & \Phi_{n}\left(d_{n}, z_{1}\right)-\Phi\left(c^{(n)}, z^{(n)}\right) \leqslant \\
\leqslant & -\frac{1}{n} \sum_{|r|<n L} d_{n}\left(\frac{r}{n}\right)\left(\bar{u}^{1}\left(\frac{r}{n}\right)-\bar{u}\left(\frac{r}{n}\right)\right)+ \\
& +\frac{1}{n} \sum_{|r|<n L}\left(\bar{u}^{1}\left(\frac{r}{n}\right)-\bar{u}\left(\frac{r}{n}\right)\right) \frac{\exp \left\{n \bar{u}_{n}^{1}(r / n)\right\}}{1+\exp \left\{n \bar{u}_{n}^{1}(r / n)\right\}}+o(1)= \\
= & \int\left(\bar{u}^{1}(x)-\bar{u}(x)\right) d x \sum_{|r|<n L}\left(\bar{u}^{1}\left(\frac{r}{n}\right)-\bar{u}\left(\frac{r}{n}\right)\right) \times \\
& \times \frac{\exp \left\{n \bar{u}^{1}\left(\frac{r}{n}\right)+n-1 / 2\left(\bar{u}\left(\frac{r}{n}\right)-\bar{u}^{1}\left(\frac{r}{n}\right)\right)+o\left(n^{-1 / 2}\right)\right\}}{1+\exp \left\{n \bar{u}^{1}\left(\frac{r}{n}\right) n^{-1 / 2}\left(\bar{u}\left(\frac{r}{n}\right)-\bar{u}^{1}\left(\frac{r}{n}\right)\right)\right\}}+o(1)= \\
= & \int_{\bar{u}^{1}(x)>0}\left(\bar{u}^{1}(x)-\bar{u}(x)\right) d x-\int_{\bar{u}^{1}(x)=0}\left(\bar{u}^{1}(x)-\bar{u}(x)\right) d x \times \\
& \times \sum_{\bar{u}^{1}\left(\frac{r}{n}\right)>0}\left(\bar{u}^{1}\left(\frac{r}{n}\right)-\bar{u}\left(\frac{r}{n}\right)\right)+o(1),
\end{aligned}
$$

где

$$
\bar{u}(x)=u(x)+z, \quad \bar{u}^{1}(x)=u^{1}(x)+z_{1} .
$$

Из последнего неравенства следует, что при $n=n_{k}$

$$
\begin{aligned}
0 \leqslant & \Phi_{n}\left(d_{n}, z_{1}\right)-\Phi\left(c^{(n)}, z^{(n)}\right) \leqslant \\
\leqslant & -\int_{\bar{u}^{1}(x)>0, \bar{u}(x)<0}(-\bar{u}(x)) \rho^{1}(x) d x- \\
& -\int_{\bar{u}^{1}(x)>0, \bar{u}(x)>0} \bar{u}(x)\left(1-\rho^{1}(x)\right) d x+o(1) \leqslant o(1) .
\end{aligned}
$$

Второе неравенство в (2.29) выполняется благодаря тому, что $\rho^{1}(x)$ удовлетворяет уравнению (2.22). Кроме того, поскольку $\Phi_{n}(c, z)$ можно представить как

$$
\Phi_{n}(c, z)=w\left(\frac{r-r^{\prime}}{n L}\right) c_{r} c_{r^{\prime}}-z+F_{n}(c, z),
$$

где $F_{n}(c, z)$ - это тоже выпуклая функция, мы получаем

$$
\begin{aligned}
\Phi_{n}(c, z)-\Phi\left(c^{(n)}, z^{(n)}\right) \geqslant & \sum_{|r|,\left|r^{\prime}\right|<n L} \frac{\partial^{2} \Phi_{n}}{\partial c_{r} \partial c_{r^{\prime}}}\left(c_{r}-c_{r}^{(n)}\right)\left(c_{r^{\prime}}-c_{r^{\prime}}^{(n)}\right)+ \\
& +\sum_{|r|<n L} \frac{\partial^{2} \Phi_{n}}{\partial c_{r} \partial z}\left(c_{r}-c_{r}^{(n)}\right)\left(z-z^{(n)}\right)+\frac{\partial^{2} \Phi_{n}}{\partial^{2} z}\left(z-z^{(n)}\right)^{2} \geqslant \\
\geqslant & \frac{2}{n} \sum_{|r|,\left|r^{\prime}\right|<n L} w\left(\frac{r-r^{\prime}}{n L}\right)\left(c_{r}-c_{r}^{(n)}\right)\left(c_{r^{\prime}}-c_{r^{\prime}}^{(n)}\right) .
\end{aligned}
$$


Итак, из (2.29) следует, что

$$
\begin{aligned}
& \frac{1}{n} \sum_{|r|,\left|r^{\prime}\right|<n L} w\left(\frac{r-r^{\prime}}{n L}\right)\left(c_{r}-c_{r}^{(n)}\right)\left(c_{r^{\prime}}-c_{r^{\prime}}^{(n)}\right)= \\
& \quad=\int \log \left|\frac{x-x^{\prime}}{L}\right|\left(\rho(x)-\rho_{1}(x)\right)\left(\rho\left(x^{\prime}\right)-\rho_{1}\left(x^{\prime}\right)\right) d x d x^{\prime}+o(1) \leqslant \\
& \leqslant \Phi_{n}(c, z)-\Phi\left(c^{(n)}, z^{(n)}\right) \leqslant o(1) .
\end{aligned}
$$

В результате мы приходим к равенствам $\rho(x)=\rho_{1}(x)$ и $z=z_{1}$.

Лемма. Если $V(x)$ удовлетворяет условиям (1.7), то существуют положительные числа $L>2$ и $\delta>0$ такие, что:

a)

$$
\left|\frac{1}{n^{2}} \log Z_{n}-\frac{1}{n^{2}} \log Z_{n}^{L}\right| \leqslant e^{-n \delta} ;
$$

б) $\partial л я\left|r_{1}\right|, \ldots,\left|r_{k}\right| \leqslant n L$

$$
\left|\rho\left(r_{1}, \ldots, r_{k}\right)-\rho_{L}\left(r_{1}, \ldots, r_{k}\right)\right| \leqslant \rho_{L}\left(r_{1}, \ldots, r_{k}\right) e^{-n \delta}
$$

в) $и \partial л я\left|r_{1}\right|>n L, u \bumpeq 10 б ъ х r_{2}, \ldots, r_{k}$

$$
\rho\left(r_{1}, \ldots, r_{k}\right) \mid \leqslant \exp \left\{-n \delta\left[V(x)-\max _{|y| \leqslant 1 / 2} V(y)\right]\right\} .
$$

ДоКАЗАТЕЛЬСТВо. Выберем $L$ настолько большим $(L>2)$, чтобы выполнялось следующее условие:

$$
V(x)-\max _{|y| \leqslant 1 / 2} V(y) \geqslant\left(2+\frac{\epsilon}{3}\right) \log |x|+\frac{\epsilon}{3}|V(x)| .
$$

Рассмотрим

$$
Z_{n}=I_{0}+\sum_{p=1}^{n} C_{n}^{p} \sum_{j_{1}, \ldots, j_{p}= \pm 1, \pm 2, \ldots} I\left(j_{1}, \ldots, j_{p}\right),
$$

где

$$
Z_{n}^{L}=\sum_{\left|r_{1}\right|, \ldots,\left|r_{n}\right| \leqslant L n} \exp \left\{-n H\left(r_{1}, \ldots, r_{n}\right)\right\}
$$

и

$$
I\left(j_{1}, \ldots, j_{p}\right)=\sum_{\left|r_{1}\right|, \ldots,\left|r_{n}\right| \leqslant L n} \exp \left\{-n H\left(r_{1}+j_{1} n L, \ldots, r_{p}+j_{p} n L, r_{p+1}, \ldots, r_{n}\right)\right\}
$$

Оценим величину

$$
\Delta\left(j_{1}, \ldots, j_{p}\right)=\log I\left(j_{1}, \ldots, j_{p}\right)-\log Z_{n}^{L}
$$


Для этого сравним каждый член в $(2.32)$ с соответствуюшим членом в (2.33). Именно для каждой конфигурации $\left|r_{p+1}\right|, \ldots,\left|r_{n}\right| \leqslant n L$ рассмотрим $\left|r_{1}^{*}\right|, \ldots,\left|r_{p}^{*}\right| \leqslant n L / 2$, которые не совпадают с какими-либо $r_{p+1}, \ldots, r_{n}$ (это возможно, т.к. $L>2$ ). Тогда получаем

$$
\begin{aligned}
-n H\left(r_{1}\right. & \left.+j_{1} n L, \ldots, r_{p}+j_{p} n L, r_{p+1}, \ldots, r_{n}\right)+n H\left(r_{1}^{*}, \ldots, r_{p}^{*}, r_{p+1}, \ldots, r_{n}\right) \leqslant \\
\leqslant & -n \sum_{1 \leqslant i \leqslant p}\left[V\left(\frac{r_{i}}{n}+j_{i} L\right)-V\left(\frac{r_{i}^{*}}{n}\right)\right]+ \\
& +2 \sum_{1 \leqslant i<k \leqslant p}\left[\log \left|\frac{r_{i}}{n}+j_{i} L-\frac{r_{k}}{n}+j_{k} L\right|-\log \left|\frac{r_{i}^{*}-r_{k}^{*}}{n}\right|\right] \times \\
& \times \sum_{1 \leqslant i \leqslant p, p<k \leqslant n}\left[\log \left|\frac{r_{i}}{n}+j_{i} L-\frac{r_{k}}{n}\right|-\log \left|\frac{r_{i}^{*}-r_{k}}{n}\right|\right] \leqslant \\
\leqslant & -n\left(2+\frac{\epsilon}{3}\right) \sum_{1 \leqslant i \leqslant p} \log L\left(\left|j_{i}\right|-\frac{1}{2}\right)+ \\
& +2 n \sum_{1 \leqslant i \leqslant p} \log L\left(\left|j_{i}\right|+\frac{1}{2}\right)+n p C+O(\log n) \leqslant \\
\leqslant & -n \frac{\epsilon}{6} \sum_{1 \leqslant i \leqslant p} \log L\left(\left|j_{i}\right|-\frac{1}{2}\right)+n p C+O(\log n) .
\end{aligned}
$$

Здесь мы воспользовались неравенством (2.31), чтобы оценить

$$
V\left(\frac{r_{i}}{n}+j_{i} L\right)-V\left(\frac{r_{i}^{*}}{n}\right)
$$

неравенством

$$
\log |a-b| \leqslant \log |a|+\log |b| \quad(|a|,|b|>1),
$$

чтобы оценить вторую сумму в правой части (2.35), а неравенством

$$
\begin{array}{r}
-\frac{1}{n} \sum_{1 \leqslant i \leqslant p, i \neq k} \log \left|\frac{r_{i}^{*}-r_{k}^{*}}{n}\right|-\frac{1}{n} \sum_{p+1 \leqslant i \leqslant n} \log \left|\frac{r_{i}-r_{k}^{*}}{n}\right| \geqslant \\
\geqslant-\frac{2}{n} \sum_{1 \leqslant i \leqslant n} \log \frac{i}{n}=-\int_{0}^{1 / 2} \ln x d x+O\left(n^{-1} \log n\right),
\end{array}
$$

чтобы оценить последнюю сумму в правой части (2.35). Подставляя последнюю оценку в (2.32), мы получаем

$$
\left|Z_{n}-Z_{n}^{L}\right| \leqslant Z_{n}^{L} n e^{-n \delta}\left(1+e^{-n \delta}\right)^{n},
$$

где $\delta$ зависит от $L$ и $\epsilon$. Этим завершается доказательство неравенства "а" леммы. Неравенства "б" и "в" могут быть доказаны аналогично. 
О СВОБОДНОЙ ЭНЕРГИИ В ДВУМЕРНОЙ КАЛИБРОВОЧНОЙ ТЕОРИИ ПОЛЯ 401

\section{Список литературы}

[1] M. Duglas, V. Kazakov. Phys. Lett. B. 1993. V. 319. P. 219.

[2] J.-M. Daul, V. Kazakov. Wilson Loop for Large $N$ Yang-Mills Theory on a Two Dimensional Sphere. Preprint LPTENS N93/37, 1993.

[3] V. Kazakov, T. Wynter. Nucl. Phys. B. 1995. V. 440. P. 407.

[4] M. L. Mehta. Random Matrices. New York: Academic Press, 1991.

[5] E. Brezin, C. Itzykson, G. Parisi, J. Zuber. Commun. Math. Phys. 1978. V. 59. P. 35.

[6] D. Bessis, C. Itzykson, J. Zuber. Adv. Appl. Math. 1980. V. 1. P. 109.

[7] A. B. de Monvel, L. Pastur, M. Shcherbina. J. Stat. Phys. 1995. V. 79. P. 585.

[8] N. I. Muskhelishvili. Singular Integral Equations. Groningen: P. Noordhoff, 1953.

[9] М.В. Щербина. ТМФ. 1989. Т. 81. С. 134.

[10] Н. Н. Боголюбов, мл. Метод аппроксимирующего гамильтониана. М.: Наука, 1971.

Поступила в редакцию 17.XI.1997 г. 